\title{
Circuit
}

Musiques contemporaines

\section{Faut-il tout accepter?}

\section{Jean-Jacques Nattiez}

Volume 1, numéro 2, 1990

Montréal musiques actuelles

URI : https://id.erudit.org/iderudit/902015ar

DOI : https://doi.org/10.7202/902015ar

Aller au sommaire du numéro

Éditeur(s)

Les Presses de l'Université de Montréal

ISSN

1183-1693 (imprimé)

1488-9692 (numérique)

Découvrir la revue

Citer cet article

Nattiez, J.-J. (1990). Faut-il tout accepter? Circuit, 1(2), 43-50.

https://doi.org/10.7202/902015ar

Ce document est protégé par la loi sur le droit d'auteur. L'utilisation des services d'Érudit (y compris la reproduction) est assujettie à sa politique d'utilisation que vous pouvez consulter en ligne.

https://apropos.erudit.org/fr/usagers/politique-dutilisation/
Cet article est diffusé et préservé par Érudit.

Érudit est un consortium interuniversitaire sans but lucratif composé de l'Université de Montréal, l'Université Laval et l'Université du Québec à Montréal. Il a pour mission la promotion et la valorisation de la recherche. https://www.erudit.org/fr/ 


\title{
Faut-il tout accepter? Jean-Jacques Nattiez
}

\author{
L'accord catégorique avec \\ l'être a pour idéal un monde \\ où la merde est niée et où \\ chacun se comporte comme \\ si elle n'existait pas. Cet idéal \\ esthétique s'appelle le kitsch.
}

Milan Kundera

Une manifestation comme Montréal Musiques Actuelles ne va pas sans soulever de graves problèmes d'ordre musical et esthétique, pour qui s'inquiète de l'état présent de la musique d'aujourd'hui et de son devenir.

On le sait: la musique contemporaine est en crise. Crise de langage, d'abord: que faire après Darmstadt, Kagel, Boulez, le minimalisme américain? Faut-il pratiquer les diverses formes du "retour à»: néoromantisme des Allemands, religiosité mystique à la Stockhausen, pratique d'une nouvelle forme de tonalité? Faut-il systématiquement, à l'âge de l' «impureté», pratiquer le métissage des cultures et le mélange des genres et des styles? Crise du public en même temps: si la musique contemporaine sérieuse survit, c'est parce qu'elle est largement subventionnée. On sait l'impact des analyses sociologiques - statistiques à l'appui - de l'ouvrage de Pierre-Michel Menger, Le Paradoxe du musicien (1983), dans le débat actuel : la musique contemporaine est une musique réservée à l'élite.

À chacun de ces deux ordres de critiques, une certaine forme (il y en a plusieurs) de postmodernisme musical répond en prenant le contrepied des impasses du modernisme. Il croyait en l'avènement d'un nouveau langage, atonal et universel. Il a échové. Prenons le parti opposé: la musique n'étant pas une question de doctrine, il n'y a pas de raisons de ne pas faire coexister John Coltrane, Michael Jackson, Stravinsky, Cage et Berio, du moment que nous y trouvons du plaisir. II n'y a plus de genres ou de styles nobles, supérieurs à d'autres. Le modernisme s'est confiné dans un monde de chapelles où prédominent les snobs; la musique d'aujourd'hui, elle, doit pouvoir s'adresser à tous les publics, en particulier aux jeunes. De 
plus, le modernisme musical vient d'Europe: opposons-lui la nouvelle musique américaine, pleine de fraîcheur et de spontanéité, qui n'a pas à défendre une tradition essoufflée et sclérosée. Le nouveau mot d'ordre semble être : plus de hiérarchies de styles, plus de hiérarchies de langages! Toutes les musiques sont bonnes, et pour tout le monde.

Montréal Musiques Actuelles me semble la parfaite illustration d'une nouvelle idéologie démagogique où un idéal quelque peu abstrait de démocratie sociale conduit, parallèlement, à la conception esthétique du : «Tout est beau, tout est bien!» Puisque nous voici à l'ère de la mondialisation médiatique et que le monde n'est plus qu'une juxtaposition de tribus qui, en principe, devraient, tout en pratiquant leurs valeurs propres, respecter les valeurs spécifiques à toutes les autres, mettons tout ensemble: un peu de gamelan et du rock heavy, de la musique électroacoustique et de l'improvisation, de la musique contemporaine "sérieuse» et des guitares électriques, la "nouvelle danse» et le multimédia, les gamineries de Ken Butler et les bondieuseries hollywoodiennes de David Borden. C'est le triomphe du kitsch! Mélangeons les styles, fusionnons les genres. Peu importe lesquels. Ils sont tous acceptables puisqu'ils sont actuels, ils témoignent de notre présent, de l'état de notre société. Nous n'avons plus les moyens d'établir entre eux la moindre hiérarchie, et, le voudrions-nous, la faillite des oukases d'un passé récent nous dit bien qu'on ne nous y reprendra plus. Et pour faire bonne mesure, quittons la salle de concert traditionnelle. Puisque le public a déserté la musique contemporaine, n'est-ce pas la musique populaire, sous ses diverses formes, qui nous indique la voie à suivre? Le Peuple, la Jeunesse ne vont pas à la Place des Arts, ce temple de la culture bourgeoise, mais dans une salle où on peut consommer de la bière pendant le spectacle, le Spectrum, ou dans un cabaret comme les Foufounes électriques, où flotte une délicieuse odeur de marijuana. Avec le choix des lieux, celui des moyens: culte du décibel et fétichisme de la sonorisation, même pour les œuvres de musique «sérieuse».

Que de bonnes intentions! Et que ne nous avait-on pas promis! Face à la sclérose de la musique européenne, Montréal devait devenir "la capitale mondiale de la création musicale»!!! On allait voir ce qu'on allait entendre! Osons donc écrire ce que beaucoup pensent tout bas, ou ce que certains des critiques qu'on vient de lire (je ne veux compromettre personne, car j'assume seul la responsabilité de ce que j'écris ici), par politesse ou discrétion, n'auront exprimé qu'en termes feutrés: pour quelques rares minutes d'extase, pour quelques moments plus forts que d'autres, que de longues plages d'ennui, que de MERDE! Et je prends le mot «merde» au sens philosophique et esthétique que lui a donné Kundera dans L'Insoutenable Légèreté de l'être, lorsque, entre autres passages, il évoque le bruit planétaire qui "fait entrer l'humanité dans la phase historique 


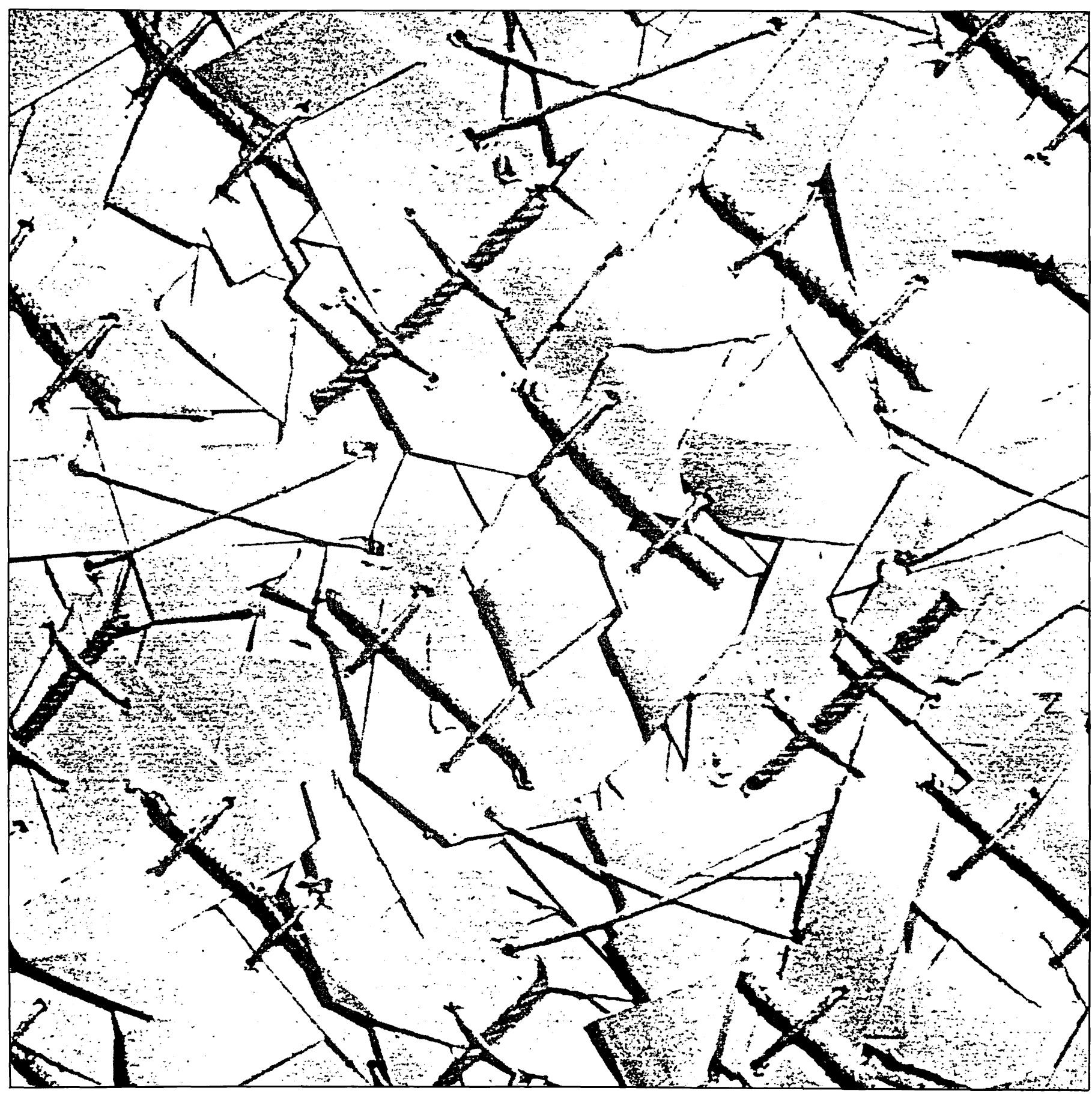


de la laideur totale» (Kundera, 1984, p. 121). Indépendamment de la somme énorme de travail exigée par son organisation, et qui force l'admiration et la reconnaissance, mais pas nécessairement l'acceptation, le festival Montréal Musiques Actuelles aura au moins eu le mérite de nous rappeler qu'au-delà des justifications bien-pensantes, la valeur n'est pas une catégorie esthétique périmée. Eł si l'on m'oppose le caractère réactionnaire de cette prise de position, je le déclare tout net: une société qui n'est plus capable d'établir ou d'admettre l'existence de hiérarchies de valeurs - i'ai bien dit, prêt à aggraver mon cas, hiérarchies - est une société mûre pour le fascisme.

Or, face aux concerts de ce festival, les jugements de valeur ne manquent pas. J'écoute une des émissions du FM de Radio-Canada qui lui a été consacrée (Musique actuelle du 11 novembre 1990). "Quels sont pour vous les moments forts du festival ? " demande Colette Mersy à trois chroniqueurs, plutôt favorables à l'événement (mais qui semblent éviter de se prononcer sur le déferlement et l'intérêt des concerts rock). Les réponses donnent à penser. Pour Michel F. Côté, le groupe ZGA de Lettonie. Pour Jean Lesage, le chef de fil de l'avant-garde minimaliste d'avant-hier, LaMonte Young, et le groupe Test Department de Grande-Bretagne. Pour Serge Provost, les deux mêmes spectacles, plus... le NEM (et les compositeurs "sérieux» américains) et la Cantate grise (Beckett, Derome, Marleau), en raison du professionnalisme du spectacle...

N'exagérons pas les échecs et les impasses des musiques "sérieuses» - je préférerais parler des «musiques contemporaines de tradition classique», si l'expression n'était pas trop lourde. Car, qu'il s'agisse, au-delà des querelles de chapelles, d'œuvres atonales, électroacoustiques ou de théâtre musical, elles nous ont apporté, ces quarante dernières années, leur contingent d'œuvres majeures, risquons le mot: de chefs-d'œuvre, dont certains sont en train de passer au statut d'œuvres classiques. II y a eu beaucoup de déchets dans la production récente, mais ni plus ni moins que dans les autres périodes de l'histoire de la musique. Nous ne connaissons plus les noms des petits Viennois moyens, des petits Mannheim moyens, et les petits Darmstadt et les petits GRM moyens seront soumis, comme à toutes les périodes historiques, au plus intransigeant des critères: le filtre de la mémoire. La sélection a déjà commencé : on entreprend déjà de rejouer ce qui apparaît bien aujourd'hui comme les œuvres majeures $\mathrm{du} X \mathrm{X}^{\mathrm{e}}$ siècle. Certains $s^{\prime} y$ emploient, avec la conviction et le talent que I'on sait. Entreprise essentielle, non seulement parce qu'il nous faut réentendre les belles œuvres, les grandes œuvres (ces mots soulignés vous irritent-ils?), mais parce qu'elles nous démontrent que, au-delà des déclarations de principe et des théories malencontreusement dévastatrices qui peuvent avoir accompagné leur naissance, il y avait là un sens de la qualité, de l'exigence, et, disons-le, un métier qui font défaut à bien des 
musiques «actuelles». Ce n'est pas n'importe quelle œuvre du passé récent que l'on rejoue.

On m'objectera sans doute: mais sur quelle base jugera-t-on de la bonne musique contemporaine? Je pourrais m'en sortir par une pirouette et dire, avec Kant, que le Beau, c'est ce qui plaît universellement sans concept, que le bon goût ne se définit pas mais qu'il se sent. Parce que cela m'entraînerait dans un long débat sur le conflit entre les universaux du goût et leur conditionnement socio-culturel, je préférerais, non pas offrir un critère, ce qui serait à coup sûr présomptueux et sans doute impossible, mais suggérer une voie de réflexion: puisque j'ose attendre de grandes œuvres, est-ce que la plupart des styles de pratique musicale qui nous ont été offerts au cours de ces onze jours de festival sont susceptibles de donner naissance à des œuvres importantes, capables de surmonter l'épreuve du temps? L'histoire récente du sérialisme et de la musique électroacoustique semble montrer que tel peut être le cas. Mais la musique répétitive? Mais les métissages rock-pop-guitare? M'inspirant ici de la réflexion du musicologue américain Leonard Meyer (1969), je crois en effet que les styles, comme les genres, possèdent différents degrés dans leur potentialité de développement et de réussite. Or, je ne crois pas que la majorité des styles de musiques pratiqués dans ce festival aient quelque chance d'engendrer un jour des chefs-d'œuvre. Je suis bien conscient de prendre ici un grand risque critique. Après tout, l'opéra résulte d'une hybridation, et on pourra m'objecter aussi qu'à l'époque postmoderne, les productions musicales plutôt que les œuvres - sont faites pour être consommées dans l'instant même de leur exécution, sans avoir à tenir compte d'un quelconque Jugement de l'Histoire! Et pourtant...! N'est-il pas arrivé au plus libéral d'entre nous de sortir d'un concert en disant: "Ça ne restera pas! Ça ne tiendra pas»? Aussi me paraît-il nécessaire d'avoir le courage de résister, sans complexes, au terrorisme des bons sentiments. Dans la justification kitsch de la MERDE musicale contemporaine, je reconnais trois arguments.

D'abord ce que j'appellerai le fatalisme sociologique. La musique-débris, le règne du scratch généralisé, l'hypostase du borborygme sonore sont acceptés, voire prônés, comme reflets de la décadence et de la violence de la société nord-américaine moderne. Est-ce une raison pour se complaire dans l'insignifiance esthétique de certaines des musiques «actuelles»? On nous fait entendre, à la radio, les improvisations pour tournedisques de Martin Tétreault et Christian Marclay (4 novembre 1990, Musée d'art contemporain). On imagine le caractère spectaculaire de la performance: devant leurs appareils et leurs piles de vieux vinyles, les «tablistes" passent d'un disque à l'autre avec virtuosité. J'écoute le résultat sonore à la radio (l'acousmatique a du bon): on s'attend à ce que musique s'ensuive. Hélas...!

Deuxième argument. Voilà la musique des jeunes, pour les jeunes. Plus de quarante ans, s'abstenir! Comme si «les Jeunes» étaient une catégorie 
globale, uniforme et indifférenciée. Qu'on ne nous fasse pas le coup de vouloir nous refiler le complexe du vieux réactionnaire décadent. Je n'accepterai jamais que I'on invoque LA Jeunesse pour servir d'alibi au manque de culture et à la médiocrité. À douze ans, la première symphonie de Mozart... À vingt-deux ans, Boulez et Le Soleil des eaux.... Exceptions, civilisation d'un autre temps, références ringardes à l'époque du vidéoclip!!! Et puis, mon cher Nattiez, vous pourriez éviter de citer Boulez une fois de plus. D'ailleurs, Daniel Caux, dans le programme du festival... Et Jean Lesage, ici même: "La France a son problème Boulez. " Mais même en lui opposant immédiatement ses ennemis intimes (Éloy, Xenakis), c'est pour aussitôt le qualifier - et je ne crois pas que ce soit par dérision de "géant de la musique»...

Pourquoi faudrait-il avoir honte, en effet, de faire référence à l'excellence, de prendre l'exemple des réussites? Mettrais-je la barre trop haut? Mais à quel âge George Benjamin commence-t-il à composer? Et Vivier attend-il longtemps pour écrire sa première œuvre vraiment originale? Je n'ai pas le sentiment de tenir des propos surannés. Chassons le bon goût, il revient au galop. On est presque gêné de rappeler des évidences banales: que la Phèdre de Pradon (vous vous souvenez de lui ?) n'arrive pas à la cheville de la Phèdre de Racine, que, quel que soit le talent d'Hergé, Les Aventures de Tintin et Milou ne méritent pas la même place, dans nos bibliothèques, que La Recherche du temps perdu, qu'entre Phil Glass et Berio, il y a la même différence qu'entre un commerçant et un orfèvre. Bref, la qualité musicale, cela existe, et la qualité ne s'évalue pas plus à coup d'indices de fréquentation des salles de concert qu'on ne distingue la qualité de la cuisine servie chez McDonald et chez Bocuse (Lyon) ou aux Mignardises (Montréal) en comptant le nombre annuel de clients accueillis chaque année.

Mais je viens d'effleurer le troisième argument des bien-pensants d'aujourd'hui. «Vous niez à la culture américaine ses caractéristiques propres!» Je n'accepte pas ce chantage à la spécificité et au relativisme culturel, car le clivage, puisque clivage il y a, ne passe pas pour moi entre l'Europe et l'Amérique, mais entre ce qui se fait de bon et de moins bon, et même de franchement mauvais, en Amérique et en Europe. D'ailleurs, c'est quoi, I' «américanité»? II va bientôt falloir ouvrir ce débat. Par exemple, répondre à la question: quelle est la part du concept de rentabilité commerciale dans l' "américanité »? Et de quels Américains parlons-nous? De Reagan ou de Woody Allen? Dois-je mettre dans le même sac, au nom de la modernité du quotidien, I'Amérique du hamburger et du Coca-Cola et celle de Hemingway, de Pollock, d'Elliott Carter ou de George Crumb? Encore des "vieux»! (Comme j'aimerais être de ces vieux-là !) Mais voilà quelques «jeunes» : je ne vois pas de nécessité de céder à la médiatisation, à la médiocrisation généralisées, sinon par lâcheté intellectuelle et par démagogie esthétique, lorsqu'une Julia Woolf ou un Tod Machover 
aux États-Unis, un Rea, un Bouliane, un Evangelista au Québec (ça y est, je cite les copains, et qui n'ai-je pas oublié de citer?!) font la preuve, influence européenne ou pas, qu'on peut avoir aussi, sur ce continent, non seulement le sens de l'œuvre bien faite, mais le talent requis pour la réussir.

Reconnaissons donc que le roi est nu. Mais pourquoi sommes-nous entourés de tant de rhinocéros? Tant pis si un jour, tel le Bérenger de lonesco, il me faudra dire: "Je suis seul, je résiste. " Mais heureusement, la rumeur insatisfaite qu'on pouvait percevoir au cours de certains entractes de Montréal Musiques Actuelles me convainc déjà que je ne résisterai pas seul. À moins que le kitsch ne submerge totalement la planète.

KUNDERA, M. (1984), L'Insoutenable Légèreté de l'être, Paris, Gallimard.

MENGER, P.M. (1983), Le Paradoxe du musicien, Paris, Flammarion.

MEYER, L.B. (1969), Music, the Arts, and Ideas, Chicago, University of Chicago Press. 


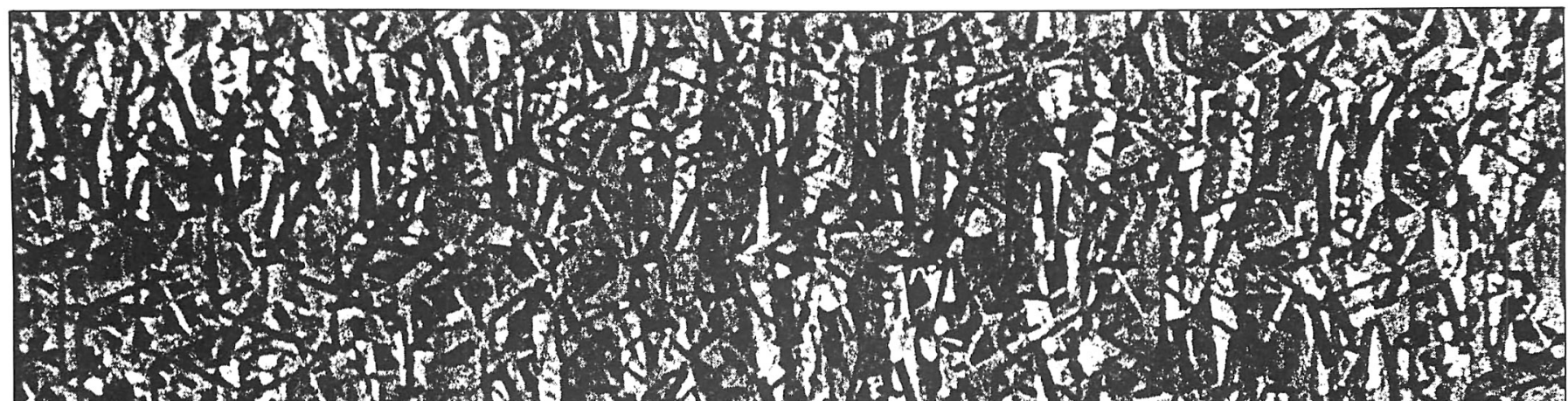
37) 7 . os.

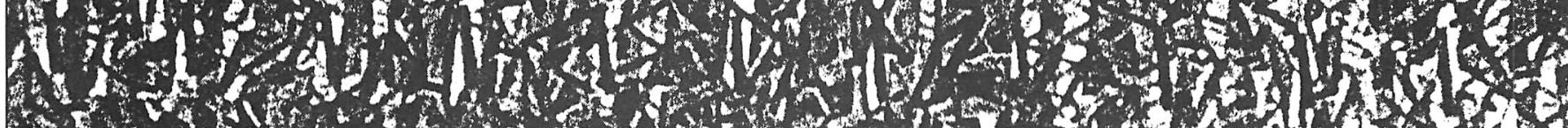

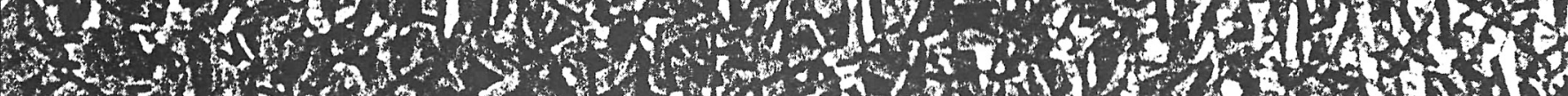

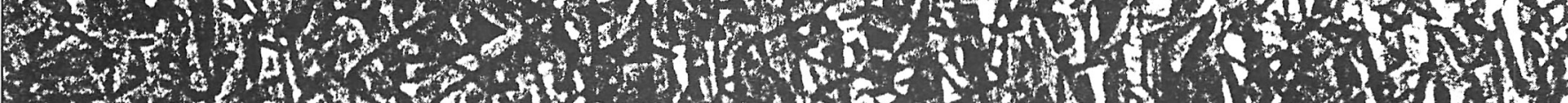
4. 15 (6) H.

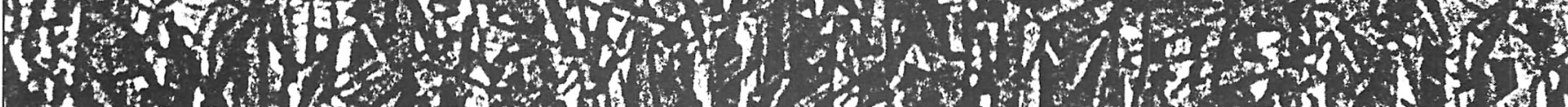

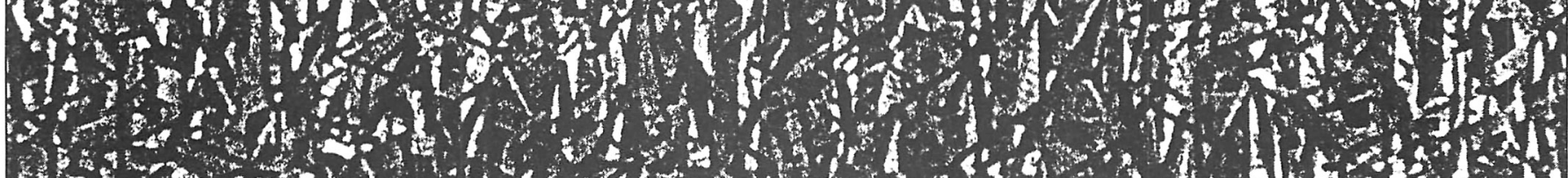

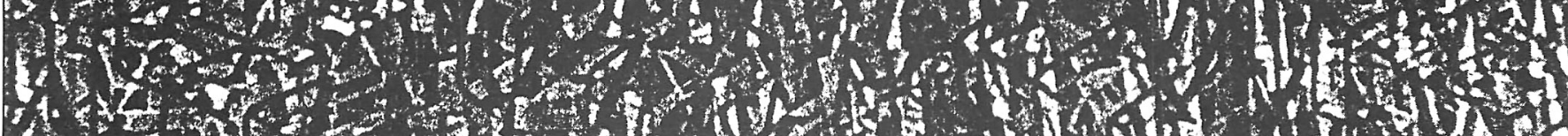
C.

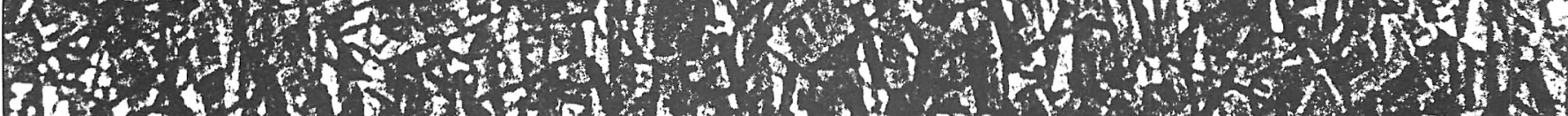
6. W

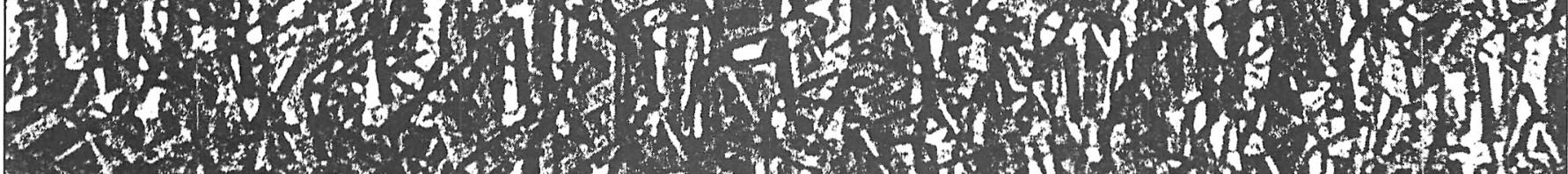
3. 